\title{
Fluoreszente chemischen Sensoren und Biosensoren in Mikrodurchflussreaktoren und miniaturisierten Elektrophoreseplattformen
}

\author{
S. Nagl, ${ }^{1}$ L. Gitlin, ${ }^{1}$ C. Herzog, ${ }^{1}$ C. Hoera, ${ }^{1}$ S. Jezierski, ${ }^{1}$ Z. Shu, ${ }^{2}$ E. Beckert, ${ }^{2}$ D. Belder ${ }^{1}$ \\ ${ }^{1}$ Institut für Analytische Chemie, Universität Leipzig, Leipzig/Deutschland, \\ ${ }^{2}$ Fraunhofer-Institut für Angewandte Optik und Feinmechanik (IOF), Jena/Deutschland \\ nagl@chemie.uni-leipzig.de
}

\section{Kurzfassung:}

Die Miniaturisierung fluoreszenter chemischer Sensoren in mikrofluidischen Kanälen in Mikrochipplattformen ermöglicht eine ortsaufgelöste, flächige Echtzeitüberwachung von Analyten und Prozessparametern im Mikromaßstab. Hier werden zwei distinkte Mikrochipplattformen mit integrierten optischen Sensoren vorgestellt.

Eine kontinuierliche und präparative Auftrennung von Proteinen und anderen Biomolekülen auf einem Mikrochip wird durch die Mikrofreiflusselektrophorese ( $\mu \mathrm{FFE}$ ) ermöglicht. Die Verfolgung von Analyt- und Trennparametern ist jedoch nur indirekt oder in nachfolgenden Schritten möglich. Basierend auf einem photolithographischen Verfahren zur Herstellung von $\mu F F E-C h i p s$ wird die Integration von fluoreszenten $\mathrm{pH}$ Sensoren in diese Mikrochips über UV Photolithographie oder Tintenstrahldruck demonstriert. Anwendungen dieser multifunktionellen Chips in der mikrofreiflussisoelektrischen Fokussierung ( $\mu$ FFIEF) von Proteinen werden gezeigt.

Mikrochip-Durchflussreaktoren sind von großem Interesse für die chemische Synthese und zur Modifikation, Bestimmung und Amplifikation von Biomolekülen und biologischer Matrizen. Wir stellen Mikrochipreaktoren mit integrierten optischen Sensoren für Sauerstoff, Temperatur und Glucose vor, die über Nassätzen, Rotations- oder Rakelbeschichtung und Softlithographie hergestellt wurden. Diese Mikroreaktoren ermöglichen die Echtzeitüberwachung dieser Parameter in chemischen und biologischen Transformationen, was hier an der metallkatalysierten Oxidation von Sulfit sowie an der enzymatischen Oxidation von Glucose demonstriert wird.

Schlüsselwörter: Miniaturisierte fluoreszente chemische Sensoren und Biosensoren, mikrofluidische freiflussisoelektrische Fokussierung, Mikroreaktor, Mikrofabrikation, multispektrale Detektion

\section{Einleitung}

Die Miniaturisierung chemischer Prozeduren in Mikrokanälen auf scheckkartengroßen Trägern, sogenannte "Lab on a chip" Systeme, hat enorme Fortschritte gemacht und ermöglicht zusätzlich zu geringerem Ressourcenverbrauch und höherer Sicherheit, Portabilität und Umweltfreundlichkeit auch eine Effizienzsteigerung vieler Verfahren durch Ausnutzung der Physik und Chemie in Mikrokavitäten.

Miniaturisierte Plattformen für chemische und biologische Synthese- und Analyseplattformen und Transformationen werden mittlerweile breit eingesetzt und sind vielfach kommerziell verfügbar. Jedoch müssen diese sehr oft noch mit anderen, im gewöhnlichen Labormaßstab durchgeführten, Prozeduren verbunden werden, wie die Kopplung mit Markern oder einer nachfolgende Analytik. Darüber hinaus erfolgt die Verfahrensoptimierung auf Mikrochips oft nach dem Prinzip von Versuch und Irrtum ohne exakte Kenntnis der Umgebungsvariablen.

Dies wollen wir verbessern, indem wir Mikrochipplattformen schaffen, die durch integrierte optische chemische Sensoren und Biosensoren Zusammensetzung und Umgebungsparameter in der Mikrofluidik in Echtzeit, räumlich aufgelöst wiedergeben.

Hierbei haben wir uns bislang auf die die Sensorintegration in miniaturisierte Trennplattformen sowie Mikrodurchflussreaktoren fokussiert. Im Bereich der Trennverfahren haben wir im Besonderen an 

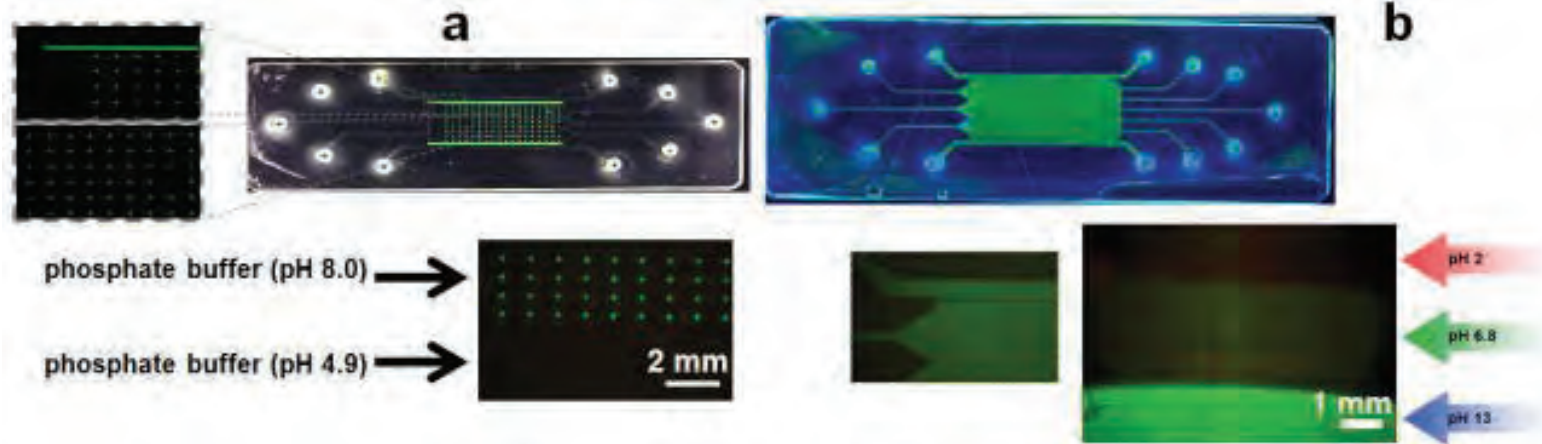

Abb. 1: Integration von fluoreszenten $\mathrm{pH}$ Sensoren in mikrofluidische Freiflusselektrophoreseplattformen über Photopolymerisation. a) pH-Sensormikroarray (Hexadecanoylaminofluorescein in Polyethylenglycol (PEG)) hergestellt über maskierte Beleuchtung, b) pH-Sensorschicht (2',7'-Bis-(2carboxyethyl)-5(6)-carboxyaminofluorescein Dextran in PEG, Funktionstest jeweils mit Phosphatpufferlösungen (40 mM) unterschiedlicher pH-Werte. Aus [1,2], Copyright Royal Society of Chemistry, 2013. Verwendet mit Erlaubnis.

der mikrofluidischen Freiflusselektrophorese $(\mu \mathrm{FFE})$ gearbeitet, welche eine milde räumliche Trennmethode für Biomoleküle darstellt, die zerstörungsfrei und präparativ angewendet werden kann. In der mikrofreiflussisoelektrischen Fokussierung ( $\mu \mathrm{FFIEF}$ ) werden Proteine, Organelle und andere Biomoleküle im nativen Zustand anhand ihres isoelektrischen Punkts (pl) aufgetrennt. Dieser kann jedoch bislang nur indirekt durch den Zusatz invasiver Substanzen oder in einem nachfolgenden Schritt quantifiziert werden. Durch die Integration von fluoreszenten $\mathrm{pH}$ Sensorschichten wird eine integrierte Analytik in der isoelektrischen Fokussierung (IEF) ermöglicht. Damit kann der pH Gradient in der IEF durch direkte Beobachtung optimiert werden und der $\mathrm{pl}$ von Proteinen und anderen Biomolekülen während der Auftrennung optisch bestimmt werden. Die Sensorintegration in $\mu F F E$ Mikrochips wurde über verschiedene Methoden basierend auf Photopolymerisation oder Tintenstrahldruck verfolgt.

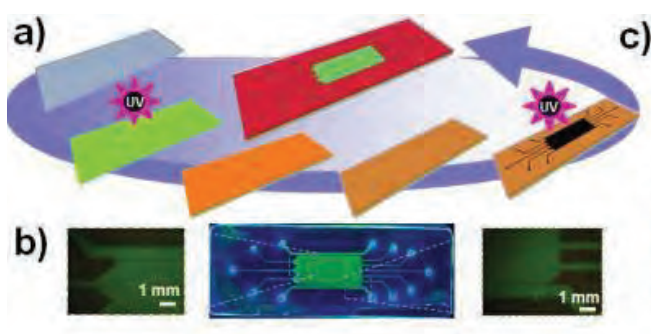

\begin{abstract}
Ergebnisse
Gezeigt in Abb. 1 sind Methoden zur photoinitierten Immobilisierung von fluoreszenten $\mathrm{pH}$ Sensoren in $\mu F F E-C h i p s$, die inrerseits durch Photopolymerisation hergestellt wurden [3]. Durch Verwendung geeigneter Photomasken wurde ein $\mathrm{pH}$ Sensormikroarray aus einem Fluoresceinderivat in einem hydrophilen PEG-Vorläufermolekül in einer $\mu F F E-S t r u k t u r$ photopolymerisiert und konnte zur optischen Verfolgung des $\mathrm{pH}$ verwendet werden (Abb. 1a). Alternativ kann in $\mu \mathrm{FFE}$ Chips eine planare $\mathrm{pH}$-Sensorschicht eingebaut werden (Abb. 1b). Ausgehend von einer flächigen $\mathrm{pH}$-Sensorschicht, die auf einem acrylierten Glasträger durch Photopolymerisation eines hydrophilen PEGs unter Einschluss fluoreszenter $\mathrm{pH}$-sensitiver Nanopartikel erzeugt wurde, wird hier in einem weiteren Schritt aus einem kurzkettigen, hydrophobem PEG Vorläufer die $\mu F F E-S t r u k t u r$ darüber photopolymerisiert (Abb. 2a, b).
\end{abstract}

Abb. 2: a) Fabrikationszyklus mikrofluidischer FFE Chips mit integrierter fluoreszenter $p H$ Sensorschicht durch multiple Photopolymerisation unterschiedlicher Oligoethylengylcole mit fluoreszenten Nanopartikeln, b) Echtfarbenfluoreszenzaufnahmen der mikrochipintegrierten Sensorschicht, c) $\mu$ FFIEF der fluoreszenzmarkierten Proteine BSA und Conalbumin (oben) mit gleichzeitiger Aufnahme des pH Gradienten (grün), Falschfarbenfluoreszenzbilder, d) Auswertung der Protein- und pH-Sensorfluoreszenzkanäle. Aus [2]. Copyright Royal Society of Chemistry, 2013. Verwendet mit Erlaubnis. 
Modellproteine Rinderserumalbumin (BSA) und Conalbumin. Diese Proteine wurden mit dem rot fluoreszierenden Marker Chromeo P503 (Py1) markiert, welcher die Ladung der Proteine nicht ändert, und auch aufgrund seiner geringen Größe nur einen vernachlässigbaren Einfluss auf den pl dieser Proteine hat. Diese Substanzen wurden im Mikrochip isoelektrisch fokussiert (Abb. 2c, oben) während gleichzeitig die grüne Fluoreszenz der $\mathrm{pH}$-Sensorschicht aufgenommen wurde (Abb. 2c, unten). Die Auswertung beider spektraler Kanäle ermöglichte die on-chip Bestimmung der plWerte beider Proteine in wenigen Sekunden. Die isoelektrischen Punkte ergaben sich zu $4.98 \pm 0.41$ (BSA) and $5.95 \pm 0.40$ (Conalbumin) was eine sehr gute Übereinstimmung zu den Literaturwerten von 4.90 und 5.88 bedeutete.

Diese Mikrochips sind demzufolge sehr vielversprechend für miniaturisierte Auftrennung von Biomolekülen mit gleichzeitiger Bestimmung ihrer Eigenschaften. integrierten $\mathrm{pH}$ Sensoren von mehreren Sekunden sowie die Beschränkung der Methode auf photopolymerisierbare Matrizen. Dies wollen wir durch Tintenstrahldrucken von $\mathrm{pH}$ Sensoren in $\mu F F E$ verbessern. Diese Methode ist ausführlich in den Proceedings dieser Konferenz zum Poster von Christin Herzog präsentiert [4].

Miniaturisierte Durchflussreaktoren ermöglichen kontinuierliche chemische Synthesen und biologische Transformationen, aber benötigen Methoden zur stetigen Überwachung und Steuerung dieser Prozesse. Wir haben Mikrochipreaktoren mit integrierten optischen Sensoren für Sauerstoff und Temperatur entwickelt, die durch kombinierte Methoden des chemischen Ätzens, verschiedene Beschichtungs- und Abtragstechniken und anschließende softlithographische Verbindung hergestellt werden (Abb. 5).

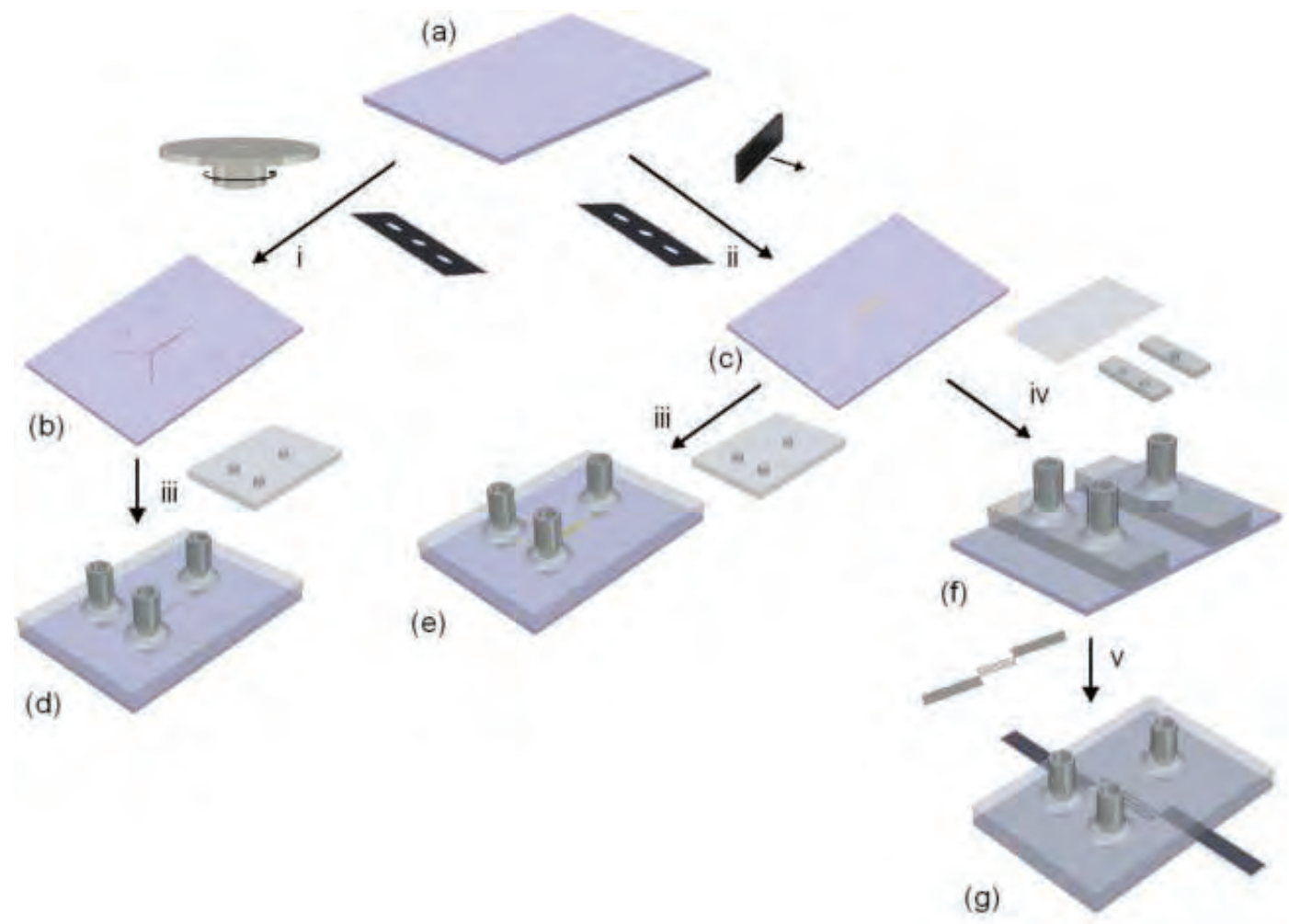

Abb. 3: Fabrikation von Mikrodurchflussreaktoren mit integrierten optischen Sauerstoff- oder Temperatursensoren, a) Nassgeätzter Glasträger mit Reaktorstruktur, b) Rotationsbeschichtung und Abtrag mit Klinge zur Integration einer Sauerstoffsensormatrix (Platinporphyrin in Polystyrol), c) Rakelbeschichtung und Abtrag mit Klinge zur Integration einer temperatursensorischen Matrix . (Ruthenium-tris-phenanthrolin in Polyacyrlonitril), d) \& e) Verbindung mit PDMS-Deckschicht mit Fluidikkontakten, f) Verbindung mit PDMS-Elementen mit Fluidikkontakten, g) Integration tintenstrahlgedruckter Mikroheizelemente (Silbernanopartikematrix) und Ausgießen in PDMS. Aus [5]. 
Rotationsbeschichtung eine Sauerstoffsensorschicht eingebracht und außerhalb der Mikrokanäle abgetragen (Abb. 3a, b). Die Mikrochips wurden mit einer Silikondeckschicht mit Fluidikanschlüssen, welche über Plasmaanwendung mit der Bodenstruktur verbunden wurde (Abb. 3d), abgeschlossen. aufgelöste Aufnahme des Sauerstoffgehalts und damit des Umsetzungsgrads der Reaktion mit hoher Sensitivität. Durch eine sensitive EMCCD Kameraaufnahme gelang eine zeitliche Auflösung von 50 ms, die Mikroreaktoren lassen sich jedoch auch mit gewöhnlichen Kameras mit Bildraten von größer als $1 \mathrm{~Hz}$ auswerten.
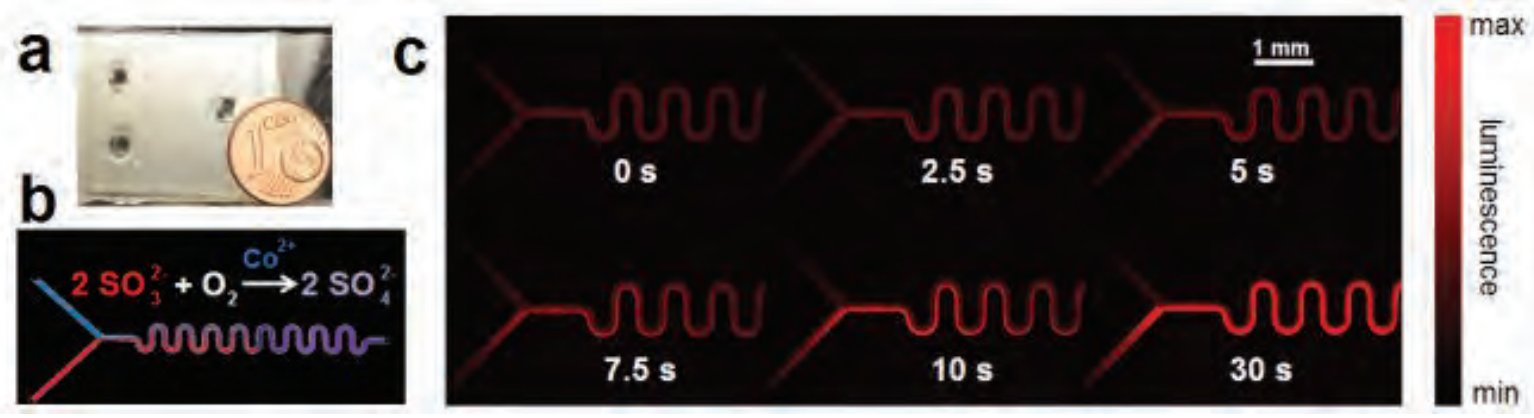

Abb. 4: Mikroreaktorchips mit integrierter Sauerstoffsensorschicht zur Echtzeitverfolgung einer metallkatalysierten Oxidation. a) Photographische Aufnahme des Mikrochips, b) Reaktionsschema der Umsetzung, c) Zeitlicher Ablauf einer Sulfitoxidation (1.5 M Natriumsulfit mit 2 mM Cobaltchlorid als Katalysator in Wasser, jeweils in den beiden Einlässen). Falschfarbenfluoreszenzaufnahmen. Aus [6]. Copyright Royal Society of Chemistry, 2013. Verwendet mit Erlaubnis.

Ein solcher Mikrochip ist in Abb. 4a gezeigt. Diese Reaktoren wurden zur Verfolgung von chemischen und biologischen Oxidationen benutzt. In $\mathrm{Abb}$. 4b ist eine schematische Darstellung einer metallkatalysierten on-chip Oxidation von Sulfit zu Sulfat gezeigt. Das Reagenz Natriumsulfit und der Katalysator Cobalt(II)chlorid wurden jeweils an einem Einlass des Mikrochips zugeführt und die Reaktion wurde über Aufnahme der Lumineszenz der Sensorschicht im roten Spektralbereich an einem mikroskopischen Aufbau nach Anregung bei $405 \mathrm{~nm}$ verfolgt (Abb. 4c)
Diese Mikrochips zeigten eine exzellente Intraund Interchip Reproduzierbarkeit der Sauerstoffsensitivität [6]. Auch biosensorische Ansätze lassen sich mit dieser Technologie realisieren. So wurden diese Sensorschichten mit den Enzymen Glucoseoxidase (GOx, $10 \mathrm{mM}$ ) und Katalase (Cat, $1 \mathrm{mM}$ ) belegt. Damit kann über Sensorik des Sauerstoffverbrauchs die Umsetzung von Glucose zu Gluconat quantifiziert werden (Abb. 5a). In Abb. 5b ist hierzu beispielhaft der räumlich aufgelöste Verbrauch einer $10 \mathrm{mM}$ Glucoselösung im Mikroreaktor bei einer Vermischung mit Pufferlösung gezeigt.
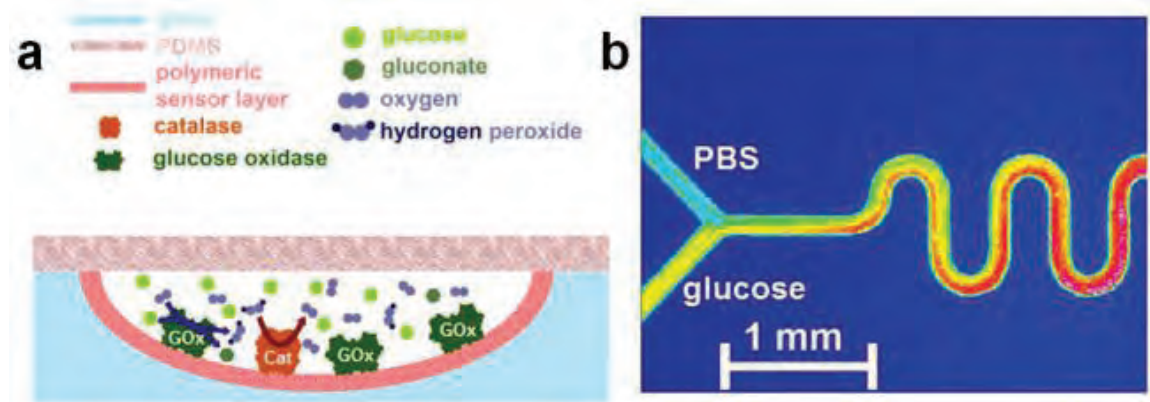

Abb. 5: Mit Glucoseoxidase und Katalase beschichteter Mikroreaktorchip mit Sauerstoffsensor zur Verfolgung einer Glucoseoxidation. a) Schematische Darstellung der Komponenten im Mikrokanal, b) Falschfarbenfluoreszenzaufnahme der Umsetzung von 10 mM Glucose im Mikroreaktor.

Wie anhand der fluoreszenzmikroskopischen Aufnahmen zu erkennen gelingt die räumliche
In einer Variation dieser Methode wurden diese Mikroreaktoren mit einem optischen 
Temperatursensor

(Ruthenium-tris-1,10 phenathrolin in Polyacrylonitril) über Rakelbeschichtung, Ausbacken und Abtrag belegt und integrierte Mikroheizer in die Reaktorchips integriert (Abb. 3c-g) und zur temperaturaufgelösten Verfolgung einer enzymatischen Umsetzung verwendet. Diese Mikrochips werden in den Proceedings dieser Konferenz zum Poster von Christian Höra präsentiert [7].

\section{Zusammenfassung}

Optische fluoreszente Sensoren ermöglichen die sensitive und selektive Echtzeitüberwachung chemischer und biologischer Analyte in miniaturisierten Synthese- und Analyseplattformen auf einem Mikrochip. Durch geeignete Fabrikations- und Detektionstechnologien können integrierte Plattformen geschaffen werden, die nachfolgende Analyseschritte überflüssig machen. Diese vielversprechenden Ansätze sollen in Zukunft noch auf andere Plattformen übertragen, zur Überwachung weiterer Spezies verwendet, sowie zur miniaturisierten und mobilen Analyse von klinisch relevanten und Umweltproben weiterentwickelt werden.

\section{Referenzen}

[1] S. Jezierski, Mikrofluidische FreiflussElektrophorese mit integrierten optischen Sensoren, Dissertation Universität Leipzig, Leipzig 2013.

[2] S. Jezierski, D. Belder, S. Nagl, Microfluidic free-flow electrophoresis chips with an integrated fluorescent sensor layer for real time $\mathrm{pH}$ imaging in isoelectric focusing, Chem. Commun. 49, 904-906 (2013); doi: 10.1039/C2CC38093E

[3] S. Jezierski, L. Gitlin, S. Nagl, D. Belder, Multistep Liquid Phase Lithography for Fast Prototyping of Microfluidic Free-FlowElectrophoresis Chips, Anal. Bioanal. Chem. 401, 2651-2656 (2011), doi: 10.1007/s00216011-5351-2

[4] C. Herzog, E. Beckert, S. Nagl, Integration fluoreszenter Mikrosensoren in mikrofluidische Elektrophoreseplattformen mittels Tintenstrahldruck und Photopolymerisation, Proceedings Dresdner Sensor-Symposium 2013.

[5] C. Höra, Mikrofluidische Reaktoren mit integrierten Mikroheizern, optischen Temperatursensoren und Sauerstoffsensoren, Masterarbeit Universität Leipzig, Leipzig 2013.
[6] L. Gitlin, C. Höra, R.J. Meier, S. Nagl, D. Belder, Micro flow reactor chips with integrated luminescent chemosensors for spatially resolved on-line chemical reaction monitoring, Lab Chip, 13, 4134-4141 (2013), doi: 10.1039/C3LC50387A

[7] C. Höra, Z. Shu, E. Beckert, S. Nagl, D. Belder, Integration fluoreszenter Temperatursensoren und resistiver Mikroheizer in mikrofluidische Durchflussreaktoren, Proceedings Dresdner Sensor-Symposium 2013. 